\title{
Integrated Rock Physics Models for Improved 4D Seismic Monitoring of CO2 Sequestration in Otway Basin, Australia
}

\author{
P.S. Wisman* (CO2CRC/Curtin University of Technology), M. Urosevic \\ (CO2CRC/Curtin University of Technology) \& T. Dance (CO2CRC/CSIRO \\ Petroleum)
}

\section{SUMMARY}

We build a detailed rock physics model that links the rock and fluid properties with seismic properties and accounts for variations in reservoir quality, fluid saturations, and seismic resolution. This approach will increase the accuracy of the predicted changes in elastic properties of the reservoir rock by calibration of the down-hole petrophysical log with core sample data and ultrasonic measurements.

The results indicate that Vp core saturated provide good agreement with $\mathrm{Vp}$ log measurement when the "effective" Kgrain is applied. Results also suggest that "effective" Kgrain may be used to represent the average mineralogy of the grains. A comparative analysis has also been performed incorporating the petrographic descriptions and geochemical analysis. The expected mineralogy derived from "effective" Kgrain and petrographic analyses are very close further confirming the model.

This type of detailed rock physics analysis provides the capability to forecast more accurately the changes in the 4D seismic response during long-term $\mathrm{CO} 2$ storage at the site, and could be a useful tool for future storage sites with similarly challenging geophysical monitoring objectives. 


\section{Amsterdam|'og}

\section{Introduction}

In most applications of Gassmann's equation, only the dry rock bulk modulus $\left(\mathrm{K}_{\mathrm{dry}}\right)$ is measured. Properties of the mineral grain modulus $\left(\mathrm{K}_{\text {grain }}\right)$ are often poorly understood and oversimplified (Batzle and Wang, 1992; Wang, 2001). $K_{\text {grain }}$ is the modulus of the solid material that includes grains, cements and pore fillings. If clays or other minerals are present with complicated distributions and structures, $\mathrm{K}_{\text {grain }}$ can vary depending on mineral composition, distribution of the grains and in-situ conditions. As a result, the calculated velocity will differ from the measured velocity.

Ultrasonic measurements of the selected core sample from reservoir rock were carried under room conditions. We obtain the dry bulk moduli $\left(\mathrm{K}_{\mathrm{dry}}\right)$ from ultrasonic measurement and derive the "effective" grain mineral moduli $\left(\mathrm{K}_{\text {grain }}\right)$ using forward Krief as a function of dry rock modulus and "exact” porosity. Then we apply Gassmann's equation to calculate lowfrequency saturated velocities from ultrasonic measurement at in-situ reservoir conditions.

The saturated velocities from ultrasonic and log measurement agree with each other when the "effective" grain mineral modulus $\left(\mathrm{K}_{\text {grain }}\right)$ is applied. Deriving the dry moduli helps us to validate the input parameters and output results of Gassmann's calculation. As a result, knowing the "effective" grain mineral modulus $\left(\mathrm{K}_{\text {grain }}\right)$ improves the accuracy of interpreting the fluid substitution thus $4 \mathrm{D}$ seismic response for $\mathrm{CO}_{2}$ sequestration monitoring program.

Here we investigate and obtain accurate prediction of elastic properties through calibration of the log and petrophysical data with core sample. To validate the calculation, we compare the core saturated velocities at in-situ reservoir condition with log measurement. We also perform a comparative analysis incorporating the petrographic and geochemical analyses. This analysis is very close further confirming the model.

\section{Methodology}

The potential error in computation of elastic properties with only logs is high unless we can use calibration with core sample. Therefore, to obtain elastic properties of reservoir rock and calibrate with field measurement, we do the following workflow in Figure 1.

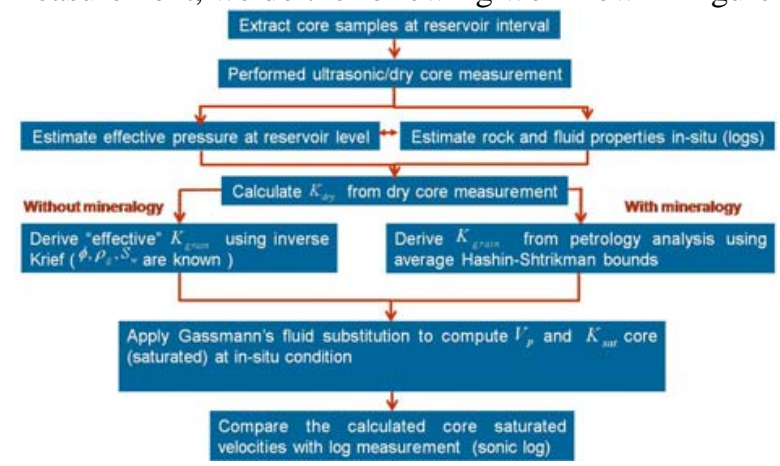

Figure 1 A methodology for computation of elastic properties.

Prediction of 4D seismic responses requires knowledge of saturation and pressure effects on seismic velocities which can be obtained by laboratory measurement using core sample (Grochau and Gurevich, 2008; Siggins, 2006). Figure 2 shows the relationship between velocity and effective pressure from ultrasonic measurement and $V_{p}$ core saturated with insitu reservoir conditions compare to $V_{p} \log$ measurement. We use Gassmann's fluid substitution to obtain low-frequency saturated velocity from ultrasonic measurement taken at in-situ condition. $\mathrm{K}_{\text {grain }}$ is derived from Krief and Hashin-Shtrikman. These computed 


\section{Amsterdaml'og}

velocities show very good agreement with the $\mathrm{V}_{\mathrm{p}} \log$ measurement when "effective" $\mathrm{K}_{\text {grain }}$ is applied which in order of $30.45 \mathrm{GPa}$. The velocity calculated differs for only $0.6-6.2 \mathrm{~m} / \mathrm{s}$ $(<0.2 \%)$ from $V_{p} \log \left(V_{p}\right.$ core saturated is $3567.4 \mathrm{~m} / \mathrm{s}$ using Krief; $V_{p}$ core saturated is 3573 $\mathrm{m} / \mathrm{s}$ using Hashin-Shtrikman; and $\mathrm{Vp} \log$ measurement is $3566.8 \mathrm{~m} / \mathrm{s}$ ). This means the elastic properties derived from ultrasonic measurement is adequate for quantitative interpretation of $4 \mathrm{D}$ seismic response.

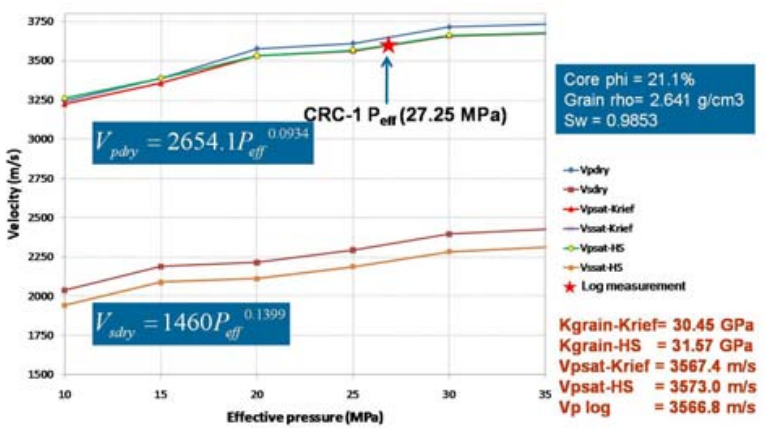

Figure 2 Velocity dependence in effective pressure for selected core sample from CRC-1 well. The different in $S$-wave velocities $\left(V_{S}\right)$ is higher than for $P$-wave $\left(V_{P}\right)$ which shows the density effect dominates.

\section{Sensivity analysis}

In practice, the measurements are always made with some errors. The problem arises when the input errors are so large that values of the calculated elastic modulus become unreliable. To gain insight into the effect of input errors onto the computation of elastic properties for this area we perform sensitivity analysis using other models. We calculate the difference in the elastic properties under in-situ reservoir condition with respect to $10 \%$ or more variations in "effective" $\mathrm{K}_{\text {grain }}$ derived from Krief, Hashin-Shtrikman, Kuster-Toksoz and Reuss. This analysis shows $10 \%$ and more variations in "effective" $K_{\text {grain }}$ produces small effect on velocity different between $\mathrm{V}_{\mathrm{p}}$ core saturated and $\mathrm{V}_{\mathrm{p}} \log$. However, estimating the "effective" $\mathrm{K}_{\text {grain }}$ accurately (with the smallest \% different) will provide high sensibility of pore fluid change and also minimize fluid substitution modelling errors, in this case is Krief (Figure 3).

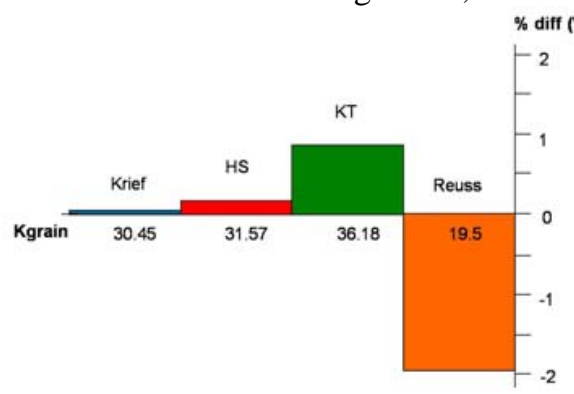

Figure 3 The different of $V_{p}$ core saturated and $V_{p} \log$ with $K_{\text {grain }}$ variations (HS is HashinShtrikman, KT is Kuster-Toksoz).

\section{Comparative analysis}

The composite log shows gamma ray, clay volume, porosity and permeability. The Waarre $-\mathrm{C}$ formation consists of 4 facies. From deeper part, tidal fluvial facies with good porosity and permeability, as we go shallow depth, gravel dominated facies with poor to moderate porosity and permeability, upper part of the Waarre-C formation where the injection taken place is amalgamated channels and transgressive sand facies with good porosity and permeability (Figure 4). Mineralogy is dominated by quartz with autogenic kaolinite as a pore-filling mineral (cement). The expected mineralogy derived from "effective” $\mathrm{K}_{\text {grain }}$ and petrographic 


\section{Amsterdaml'og}

analyses are very close further confirming the model where $\mathrm{K}_{\text {grain }}$ is "soft" ( $\mathrm{K}_{\text {grain }}$ from Krief is $30.45 \mathrm{GPa}$ ). The softer the rock frame, the bigger the elastic properties changes. This is a first-order requirement for successful 4D seismic monitoring (Wang, 1997).

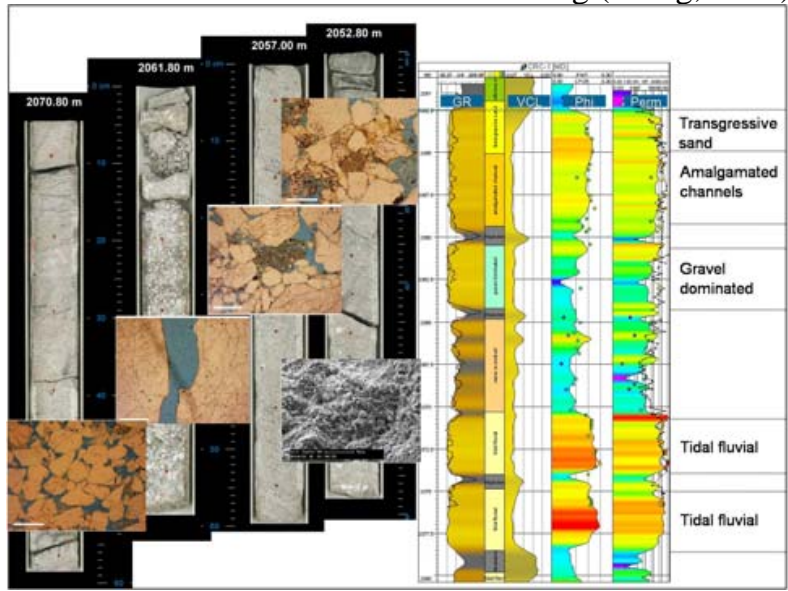

Figure 4 Composite log of CRC-1 well incorporating with petrographic thin section. 4 facies are defined within Waarre-C formation.

\section{Results}

Initial forward modelling of the 4D seismic response predicted at Naylor-1 and CRC-1 well that only a 1-3\% change in elastic properties could be expected (Li et al., 2006; Wisman and Urosevic, 2007). After applying the "effective” $\mathrm{K}_{\text {grain, }}$ elastic properties changes for CRC-1 well after fluid substitution show greater changes in $V_{P}$, and significant changes in AI (Acoustic impedance) which is driven by changing in density. If the impedance changes greater than 4\%, the change in seismic response is detectable (Lumley, 2001)The same methodology is applied for Naylor-1 well (monitoring well) and shows greater changes compare to the preliminarily modelling as expected (Figure 5 and 6).

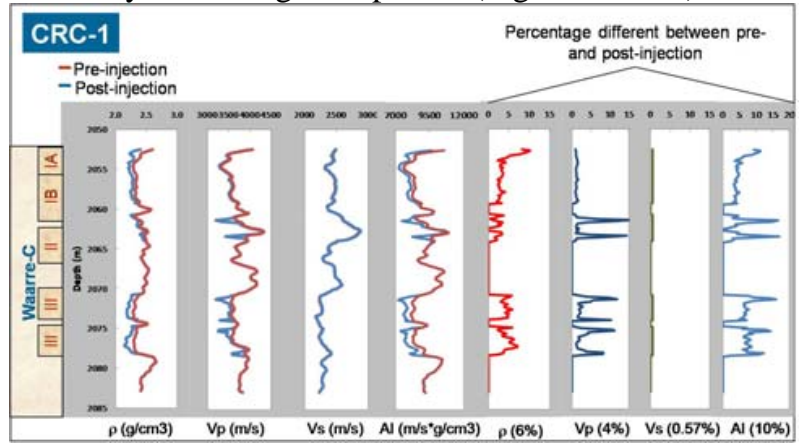

Figure 5 Fluid substitution modelling for CRC-1 well.

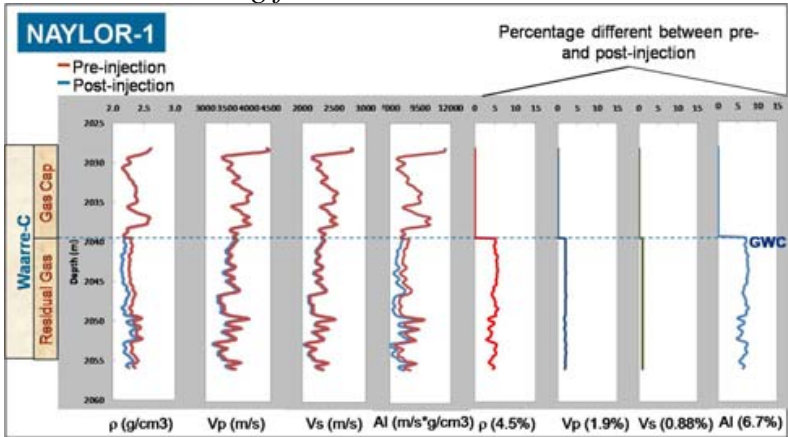

Figure 6 Fluid substitution modelling for Naylor-1 well. 


\section{Amsterdam|'og}

\section{Conclusions}

We describe a methodology for accurate prediction of elastic properties of the reservoir rock through calibration of the log and petrophysical data with core sample. We propose forward Krief to be used to obtain "effective" $K_{\text {grain. }}$ "Effective" $K_{\text {grain }}$ represents the average mineralogy if the composition is unknown. Applying "effective" $K_{\text {grain }}$ with fluid substitution will result in core saturated velocity agreeing with log measurement hence further confirming the model. As a result, the impedance change is greater than 4\%, hence 4D seismic responses are detectable. The later can guide modelling and inversion process.

The rock physics models provide the capability to forecast more accurately the changes in the $4 \mathrm{D}$ seismic response during long-term $\mathrm{CO}_{2}$ storage and could be useful tool for future storage sites with similarly challenging geophysical monitoring objectives.

\section{Acknowledgements}

We thank CO2CRC for funding the research project, permission to publish this paper and PhD scholarship for Putri Wisman. We are also grateful to Tony Siggins, Ulrike Schacht, and Otway Project team members for providing the data and useful discussion.

\section{References}

Batzle, M. And Wang, Z. [1992] Seismic properties of pore fluids. Geophysics, 57, 11, 13961408.

Grouchau, M.H. and Gurevich, B. [2008] Investigation of core data reliability to support timelapse interpretation in Campos Basin, Brazil. Geophysics, 73, 2, E59-E65.

Li, R., Urosevic, M. And Dodds, K. [2006] Prediction of 4D seismic responses for the Otway Basin CO2 sequestration site. $76^{\text {th }}$ SEG International Exposition and Annual Meeting, Extended Abstracts, 25, 1, 2181-2185.

Lumley, D.E. [2001] The next wave in reservoir monitoring: The instrumented oil field. The Leading Edge, 20, 6, 640-648.

Siggins, A.F. [2006] Velocity-effective stress response of CO2-saturated sandstones. Exploration Geophysics, 37, 60-66.

Wang, Z. [1997] Feasibility of time-lapse seismic reservoir monitoring: The physical basis. The Leading Edge, 16, 9, 1327-1330.

Wang, Z. [2001] Fundamental of seismic rock physics. Geophysics, 66, 2, 398-412.

Wisman, P. and Urosevic, M. [2007] Geophysical modelling comparison at varying saturation and pressure: CO2 sequestration pilot project at Otway Basin. $19^{\text {th }}$ ASEG Conference and Exhibition, Extended Abstract. 